\title{
RESEARCH
}

Open Access

\section{Early steroid pulse therapy among children with influenza virus-associated encephalopathy}

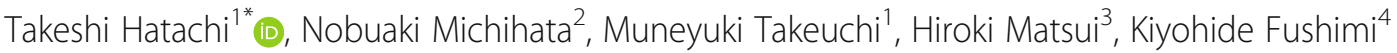
and Hideo Yasunaga ${ }^{3}$

\begin{abstract}
Background: Influenza virus-associated encephalopathy (IAE) can lead to neurological sequela and mortality among children. Therefore, instant recognition and therapeutic intervention for IAE are crucial. In some clinical subtypes of IAE, steroid pulse therapy might be beneficial, especially when it is administered in the early phase. However, early identification of patients who may benefit from steroid pulse therapy is sometimes difficult. We aimed to assess the effectiveness of early steroid pulse therapy among children with IAE.

Methods: In this retrospective observational study, we used a national database that covers half of the acute care inpatients across Japan to identify inpatients aged $\leq 18$ years with a diagnosis of IAE between July 2010 and March 2017. Unfavorable outcome was defined as a composite outcome of sequela including Japan Coma Scale $\geq 10$ at discharge, requiring tracheostomy, mechanical ventilation, enteral tube feeding, rehabilitation at discharge, or inhospital death. Propensity score matching was performed to compare unfavorable outcome and in-hospital mortality between patients with and without steroid pulse therapy within 2 days of admission.

Results: Among 692 patients included in the study, the mean age was 5.8 years, and 55.8\% were male. The overall in-hospital mortality was $1.3 \%$, and the proportion of the unfavorable outcome was $15.0 \%$. We observed no significant difference in the unfavorable outcome between matched patients (168 patients in each group) with and without early steroid pulse therapy $(13.7 \%$ vs $8.3 \%$; $P=0.16)$ or in-hospital mortality $(0.6 \%$ vs $1.2 \% ; P=1.0)$.

Conclusions: We did not observe the effectiveness of early steroid pulse therapy on patient outcomes among children with IAE in our study population including all clinical subtypes of IAE. Further studies considering severity of illness are warranted to determine whether steroid pulse therapy is beneficial, especially for specific clinical subtypes of IAE.
\end{abstract}

Keywords: Influenza, Encephalopathy, Encephalitis, Steroids, Glucocorticoids, Pediatrics

\footnotetext{
* Correspondence: hatachi@wch.opho.jp

'Department of Intensive Care Medicine, Osaka Women's and Children's

Hospital, 840 Murodocho, Osaka, Izumi 594-1101, Japan

Full list of author information is available at the end of the article
}

(C) The Author(s). 2020 Open Access This article is licensed under a Creative Commons Attribution 4.0 International License, which permits use, sharing, adaptation, distribution and reproduction in any medium or format, as long as you give appropriate credit to the original author(s) and the source, provide a link to the Creative Commons licence, and indicate if changes were made. The images or other third party material in this article are included in the article's Creative Commons licence, unless indicated otherwise in a credit line to the material. If material is not included in the article's Creative Commons licence and your intended use is not permitted by statutory regulation or exceeds the permitted use, you will need to obtain permission directly from the copyright holder. To view a copy of this licence, visit http://creativecommons.org/licenses/by/4.0/ The Creative Commons Public Domain Dedication waiver (http://creativecommons.org/publicdomain/zero/1.0/) applies to the data made available in this article, unless otherwise stated in a credit line to the data. 


\section{Background}

Acute encephalopathy, one of the most serious complications among children with influenza virus infection, can lead to neurological sequela and mortality [1]. Neurological symptoms and death occur very early from the onset of influenza symptoms [2]. Instant recognition and intervention for influenza virus-associated encephalopathy (IAE) are therefore crucial.

IAE has been reported worldwide [3-7], but most frequently in Japan [8-10]. This implies difference in genetic background of patients between Japan and other countries [11]. IAE is generally classified according to clinical subtypes including clinically mild encephalitis/ encephalopathy with a reversible splenial lesion (MERS), acute encephalopathy with biphasic seizures and late reduced diffusion (AESD), and acute necrotizing encephalopathy (ANE) [8]. In some IAE cases, increased levels of cytokines in the serum or cerebral fluid were observed [12-14], and immunomodulatory therapy such as steroid pulse therapy was therefore proposed and is widely used in addition to systemic support and anti-influenza agents [15-17].

Some previous studies reported that only "early" steroid pulse therapy could be beneficial among children with IAE $[15,17]$, while most frequent clinical IAE subtypes such as MERS had favorable outcomes without aggressive therapeutic interventions including steroid pulse therapy [8]. However, identification of clinical subtype of IAE or prediction of patient prognosis are sometimes difficult in the early phase when steroid pulse therapy is thought to be beneficial $[18,19]$. Therefore, it is necessary to assess whether early steroid pulse therapy is beneficial among children with IAE as a whole.

In this study, we aimed to assess the effectiveness of early steroid pulse therapy on patient outcomes among children with all subtypes of IAE using a nationwide database in Japan.

\section{Methods}

\section{Study design}

This was a retrospective observational study using a nationwide database.

\section{Database}

All data were retrospectively abstracted from the Diagnosis Procedure Combination database in Japan. This database is a national inpatient database that includes administrative claims and discharge abstract data from more than 1000 participating acute care hospitals across Japan. All academic hospitals are obliged to contribute to the database, and community hospitals participate voluntarily. Approximately $50 \%$ of all acute-care inpatient data of all ages in Japan are included in the database. The details of this database were described in a previous study [20].

\section{Participants}

Patients aged $\leq 18$ years who were admitted to hospitals with a diagnosis of IAE and discharged between July 2010 and March 2017 were included in the study. Diagnosis of IAE was identified according to documented diagnosis of influenza virus infection and encephalopathy during the same hospitalization. Diagnosis of influenza virus infection was determined according to the International Classification of Diseases 10th Revision (ICD-10) codes of 09 (influenza due to identified zoonotic or pandemic influenza virus), J10 (influenza due to identified seasonal influenza virus), and J11 (influenza, virus not identified), as well as a manually documented diagnosis of influenza virus infection. Diagnosis of encephalopathy was determined according to diagnostic codes including virus-associated encephalopathy (ICD10 code: G948), acute encephalopathy (G934), and delirium due to other medical condition (F058). When a patient was admitted twice or more to the same hospital with a diagnosis of IAE during the study period, only the first admission with a diagnosis of IAE was included.

We excluded the following patients: (1) those transferred from other hospitals because their therapeutic interventions at the initial hospital were unknown, (2) those transferred to other hospitals within 7 days of admission because their outcomes were difficult to determine, (3) those who died within 2 days of admission to avoid immortal time bias, and (4) those aged $<1$ month at admission to avoid including unproved congenital diseases such as metabolic disorders.

\section{Outcomes}

Unfavorable outcome was defined as a composite outcome of neurologic sequela and death during the hospital stay including (1) the Japan Coma Scale (JCS) $\geq 10$ at discharge, (2) requiring tracheostomy during hospital stay, (3) requiring mechanical ventilation at discharge, (4) requiring enteral tube feeding at discharge, (5) requiring rehabilitation at discharge, and (6) in-hospital death. These unfavorable outcomes did not include those that already existed before admission with IAE.

JCS comprises four categories: alert, 1-digit (awake without stimulation), 2-digit (awake with stimulation), and 3-digit codes (non-awake with stimulation) [21]. Each category has subcategories (1, 2, 3 in the 1-digit code; 10, 20, 30 in the 2-digit code; and 100, 200, 300 in the 3 -digit code). JCS 10 indicates that a patient did not open his/her eyes spontaneously; however, he/she opened them in response to a normal volume of voice. 


\section{Therapeutic interventions}

Anti-influenza agents included peramivir hydrate, oseltamivir phosphate, laninamivir octanoate hydrate, and zanamivir hydrate. Intravenous antiepileptic drugs included thiopental, thiamylal, propofol, midazolam, diazepam, fosphenytoin, phenytoin, phenobarbital, and levetiracetam. Vasoactive agents included adrenaline, noradrenaline, dopamine, and dobutamine. Steroid pulse therapy was generally administered for 3 consecutive days and comprised $30 \mathrm{mg} / \mathrm{kg}$ bodyweight of intravenous methylprednisolone per day [16]. We defined steroid pulse therapy as intravenous administration of any steroid equivalent to methylprednisolone $\geq 30 \mathrm{mg} / \mathrm{kg}$ body weight during the hospital stay that was initiated within 2 days of admission.

\section{Covariates}

We collected patient demographic data such as age, sex, body weight at admission, and data on the hospitals they were admitted to. Congenital anomaly was detected according to the ICD-10 codes Q00-99, and epilepsy was detected according to the ICD-10 codes G40 and G41. Epilepsy did not include patients diagnosed after the incidence of IAE. We collected the JCS at admission and at discharge, tracheostomy, mechanical ventilation, enteral tube feeding, and rehabilitation during the hospital stay. Patients with missing data were excluded. We also collected the in-hospital mortality rate of patients, date of admission and discharge, and therapeutic interventions described above.

\section{Statistical methods}

Categorical variables were evaluated using Fisher's exact test. Continuous variables were evaluated using the Student's $t$ test. Propensity score matching was performed with 1:1 nearest-neighbor matching without replacement using a caliper width of 0.2 of the pooled standard deviation of the logit of the propensity scores between patients with and without steroid pulse therapy [22]. The following factors were included to estimate propensity scores: age category ( $<1$ year, $1-5$ years, $6-11$ years, $12-$ 18 years), sex, congenital anomaly, epilepsy at admission, JCS category at admission (0, 1-3, 10-30, and 100-300), and the following factors that were implemented within 2 days of admission: anti-influenza agents, intravenous antiepileptic drugs, acetaminophen, mechanical ventilation, vasoactive agents, computerized tomography or magnetic resonance imaging, cerebrospinal fluid examination, admission to intensive care unit, and immunoglobulin. We calculated absolute standardized differences to check the balance in the distributions of these factors in the unmatched and propensity scorematched groups with and without steroid pulse therapy. Absolute standardized differences of $<10 \%$ are generally considered negligible imbalances [23]. We then compared the patient outcomes between the propensity score-matched groups with and without steroid pulse therapy using Fisher's exact test. A post hoc sensitivity analysis was performed using propensity score matching for patients with an ICD-10 code of G948 (virus-associated encephalopathy) or G934 (acute encephalopathy) and excluding those with code F058 only (delirium due to other medical condition). Statistical significance was defined when two-sided $P$ values were $<0.05$. All analyses were conducted using Stata version 15.0 (Stata Corp LLC, College Station, Texas).

This study followed the Strengthening the Reporting of Observational Studies in Epidemiology (STROBE) reporting guideline [24] (Supplementary Data).

\section{Results}

\section{Study population}

During the study period, 821 patients aged $\leq 18$ years were admitted to hospital with a diagnosis of IAE and discharged. Among these, 106 patients were transferred from other hospitals, 22 patients were transferred to another hospital within 7 days of admission, and 3 patients died within 2 days of admission. Two patients were transferred from other hospitals and were transferred to other hospitals. There was no neonatal patient aged $<1$ month. Consequently, 692 patients were included in the analysis (Fig. 1).

Among those included in the analysis, the mean age was 5.8 years (95\% confidence interval (CI), 5.5-6.1), 386 (55.8\%) patients were male, $8(1.2 \%)$ patients had a congenital anomaly, and 115 (16.6\%) patients had preexisting epilepsy at admission. These 692 patients were cared for in 261 hospitals across Japan. The mean length of stay was 13.6 days $(95 \% \mathrm{CI}, 11.4-15.8)$. The overall inhospital mortality was 15 out of 821 (1.8\%) among all children with IAE identified in the database and 9 out of $692(1.3 \%)$ among patients included in the analysis.

\section{Patient characteristics and therapeutic interventions}

Table 1 shows the patient characteristics and therapeutic interventions among unmatched patients with and without early steroid pulse therapy. Among 329 patients with steroid pulse therapy $(\geq 30 \mathrm{mg} / \mathrm{kg}$ ) initiated within 2 days of admission, $235(71.4 \%)$ received $\geq 90 \mathrm{mg} / \mathrm{kg}$ of steroids. The median initiation day of steroid pulse therapy was 1.7 days (95\% CI, 1.6-1.9). Table 1 also shows the characteristics and therapeutic interventions administered within 2 days of admission between propensity score-matched patients with and without early steroid pulse therapy. Absolute standardized differences were $\leq$ $10 \%$ for most of the characteristics, except for epilepsy $(11.6 \%)$. There were no missing data in the database regarding the variables included in Table 1. 


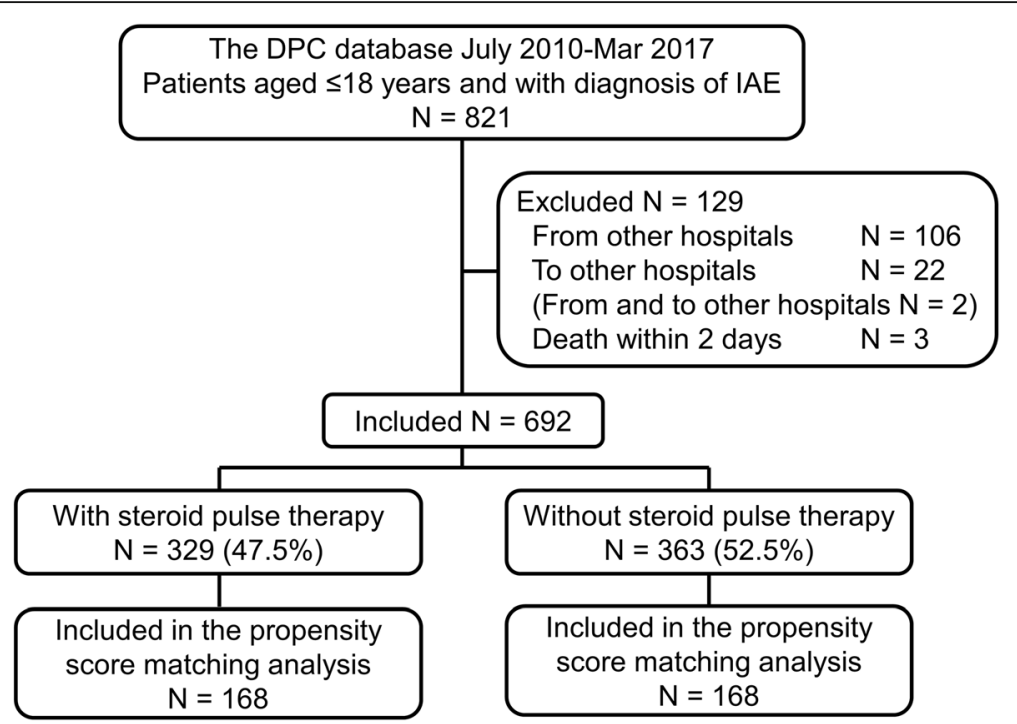

Fig. 1 Flowchart of patients in the study. DPC database Diagnosis Procedure Combination database, IAE influenza virus-associated encephalopathy. On the basis of the International Classification of Diseases 10th Revision, 231 (33.4\%) patients out of 692 patients included in the study had the G948 code (virus-associated encephalopathy), 429 (62.0\%) had the G934 code (acute encephalopathy), and 45 (6.5\%) had the F058 code (delirium due to other medical condition) including duplications. Of these, 11 patients had G948 and G934 codes, 1 had G948 and F058 codes, and 1 had G934 and F058 codes

\section{Clinical endpoint}

Among 692 patients included in the analysis, the number of patients with each unfavorable outcome is shown in Table 2. There were $104(15.0 \%)$ patients with the composite unfavorable outcome. The mean length of stay was 8.2 days (95\% CI, 7.7-8.8) and 43.7 days (95\% CI, 30.6-56.8) among patients with favorable and unfavorable outcomes, respectively $(P<0.001)$. There were no missing data in the database regarding the outcome variables included in Table 2 except for JCS scores for deceased patients; therefore, none of the patients were excluded from the analyses.

\section{Effectiveness of early steroid pulse therapy}

Table 3 shows patient outcomes between propensity score-matched patients with and without early steroid pulse therapy. There was no significant difference in the proportions of the unfavorable outcomes between matched patients with and without early steroid pulse therapy $(13.7 \%$ vs $8.3 \%$; $P=0.16)$ or in-hospital mortality (0.6\% vs $1.2 \% ; P=1.0)$.

When performing a post hoc sensitivity analysis by propensity score matching for patients with an ICD-10 code of G948 (virus-associated encephalopathy) or G934 (acute encephalopathy) $(N=649)$, the proportions of the composite unfavorable outcomes were 26 out of 166 (15.7\%) and 17 out of $166(10.2 \%)$ in patients with and without early steroid pulse therapy, respectively $(P=0.19)$. In this sensitivity analysis, in-hospital mortalities were 0 out of
$166(0 \%)$ and 2 out of $166(1.2 \%)$ in patients with and without early steroid pulse therapy, respectively $(P=0.50)$.

\section{Discussion}

In this retrospective observational study, we identified 821 pediatric patients admitted to hospital with a diagnosis of IAE during seven influenza seasons between 2010 and 2017. Of these, we included 692 eligible patients in the analysis and identified that the mortality rate was $1.3 \%$, and the unfavorable rate was $15.0 \%$. We observed no significant difference in patient outcomes between the propensity score-matched patients with and without early steroid pulse therapy in our study population, including all clinical subtypes of IAE.

IAE is generally diagnosed when a patient with influenza virus infection develops neurological symptoms such as delirious behavior, seizure, vomiting, consciousness disorder, and coma $[10,25]$. These neurological symptoms usually occur within a few days of influenza virus infection symptoms such as fever, rhinitis, and cough [2]. Although, there are no established diagnostic criteria, we included patients who were clinically diagnosed with IAE by physicians at each hospital and identified those patients according to ICD-10 codes and documented diagnosis in the database.

In our study population, the mean and median ages were 5.8 years and 4.5 years, respectively (the most frequent age was 1 year), and $55.8 \%$ were male, similar to previous studies [8, 25]. Additionally, a previous study 
Table 1 Patient characteristics and interventions among unmatched and propensity score-matched patients with and without steroid therapy

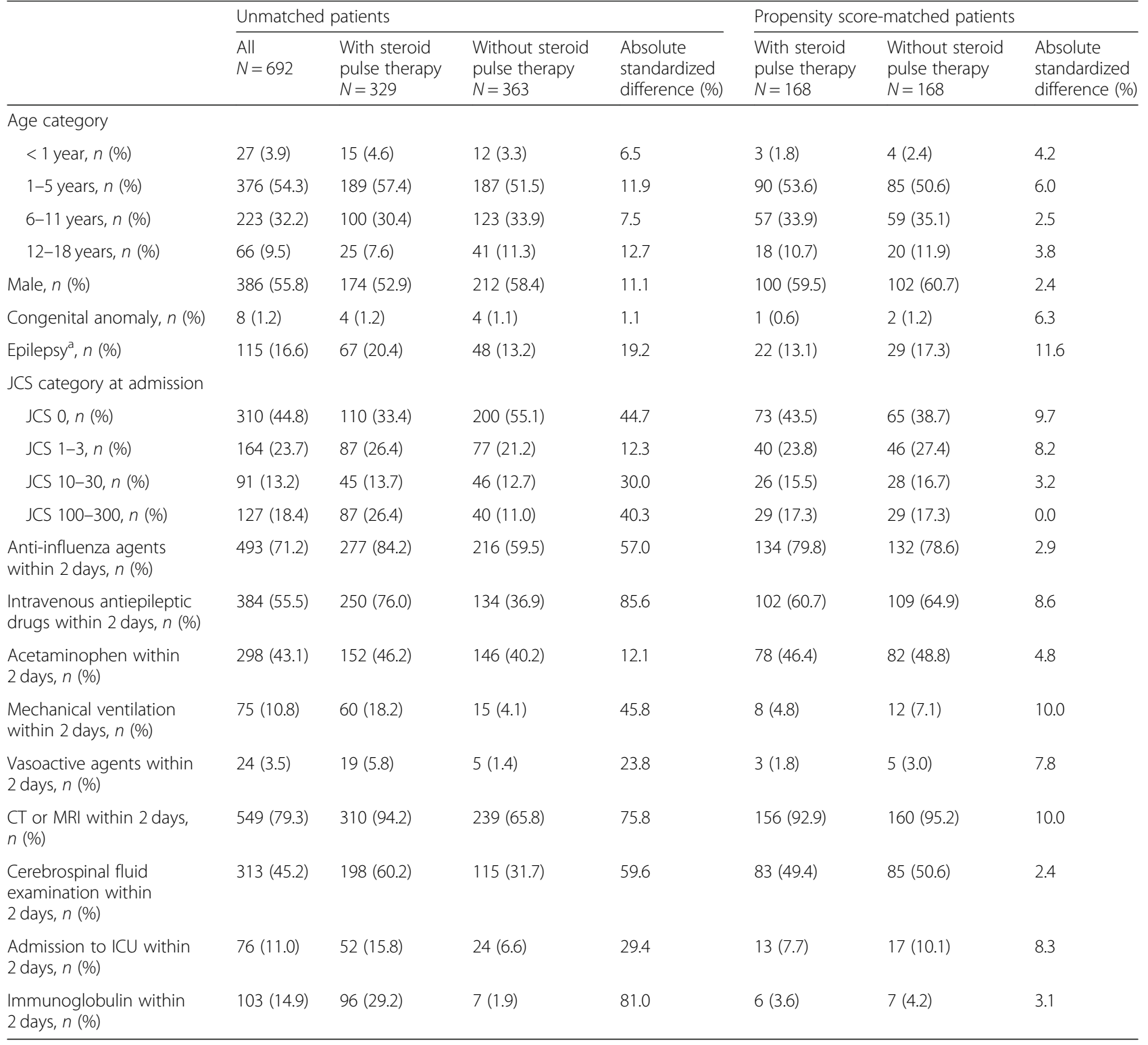

JCS Japan Coma Scale, CT Computerized tomography, MRI Magnetic resonance imaging, ICU Intensive care unit

aEpilepsy patients did not include those diagnosed after the incidence of influenza virus-associated encephalopathy

reported that $84.5 \%$ of children with IAE had no underlying disease before the onset of IAE [25], which was similar to our study $(82.7 \%$ without congenital anomaly or epilepsy). Therefore, most of the patients with IAE were previously healthy children.

Among IAE as a whole, in-hospital mortality was as high as $31.8 \%$ in a questionnaire study from 1998 to 1999 [25]; thereafter, it improved to $6.8 \%$ in another questionnaire study between 2007 and 2010 [8], was $7.8 \%$ in a national surveillance database study between 2010 and 2015 [10], and was $1.8 \%$ in our study between 2010 and 2017. Similarly, the proportions of patients with neurologic sequela or mortality were reported as $59.5 \%$ in $1998-1999$ [25], which improved to $23.6 \%$ in 2007-2010 [8] and $15.0 \%$ in 2010-2017 in our study population. The differences in mortality and proportions of unfavorable outcomes may be explained by recent improvements in patient outcome among children with IAE [8], differences in case definition and severity of IAE, reporting bias according to the study design, and differences in the definition of unfavorable outcome. However, although patient outcomes including sequela and mortality have improved recently, they remain unacceptable. 
Table 2 Number of patients with each unfavorable outcome

\begin{tabular}{ll}
\hline Outcomes & Number of patients (\%) \\
\hline Composite unfavorable outcome & $104(15.0)$ \\
In-hospital death & $9(1.3)$ \\
$J C S \geq 10$ at discharge ${ }^{a}$ & $21(3.0)$ \\
Tracheostomy during hospital stay & $8(1.2)$ \\
Mechanical ventilation at discharge & $15(2.2)$ \\
Enteral tube feeding at discharge & $21(3.0)$ \\
Rehabilitation at discharge & $83(12.0)$ \\
\hline
\end{tabular}

Seventy-eight patients had one unfavorable outcome; twelve patients had two; six patients had three; four patients had four; three patients had five; and one patient had six.

JCS Japan Coma Scale

ancluding deceased patients

IAE includes variety of clinical manifestations such as AESD, ANE, and MERS $[1,8]$. In patients with IAE, brain dysfunctions are thought to be caused by cerebral edema [1]. Although the pathogenesis of IAE is poorly understood, it may involve viral invasion to the central nervous system, inflammatory cytokines, metabolic disorders, and genetic susceptibility [26]. Influenza virus is rarely detected in blood or cerebrospinal fluid samples, which suggests direct invasion of the virus into the central nervous system, although this is uncommon [27]. However, increased cytokine levels in serum or cerebral fluid were observed among children with IAE [12-14]. Although chemokines and proinflammatory cytokines play essential defense roles against influenza virus [28], exuberant immune responses with excessive levels of them described as a "cytokine storm" may lead to extra pulmonary complications including encephalopathy [29]. Therefore, in some clinical subtypes of IAE, immunomodulatory therapies to mitigate the systemic cytokine storm have been proposed. These therapies include steroid therapy [15-17], immunoglobulin [16, 17], cyclosporine [16], and plasma exchange [16]. Among these therapies, steroid therapy in the form of methylprednisolone steroid pulse therapy is documented in the Japanese clinical guidelines for IAE [16] and widely used in Japan [15]. However, such an immunosuppression therapy in patient with infections might lead to more virulent illness, and evidence for these treatments are not sufficient [1]. Therefore, we aimed to assess the effectiveness of steroid pulse therapy among children with IAE using a nationwide database in Japan.
In our study population including all clinical subtypes of IAE, after adjusting for the factors in Table 1, we did not observe a significant difference in the proportions of unfavorable outcomes or in-hospital mortality between the patients with and without early steroid pulse therapy within 2 days. The effectiveness of steroid pulse therapy among children with IAE has not been assessed previously in randomized controlled studies. A previous descriptive study identified the wide use of steroid pulse therapy (39.2\%) for IAE in Japan [15]. They observed lower incidences of severe disability or death among children who received "earlier" steroid pulse therapy: 0 out of 9 patients who received steroid pulse therapy within 1 day of the onset of IAE had severe disability or death, 5 out of 11 patients who received therapy on the second day had severe disability or death, and 7 out of 9 patients who received therapy on or after the third day had severe disability or death. However, as described by the authors, the severity of illness at admission was not adjusted for in their study. Another previous study investigated patients with ANE without brain stem lesions ( $N=17$; influenza related, $N=5$ ), and observed a lower incidence of unfavorable outcomes among patients who received early steroids: 5 out of 12 patients who received early steroids (within $24 \mathrm{~h}$ ) had unfavorable outcomes, and 5 out of 5 patients who received steroids after $24 \mathrm{~h}$ or who did not receive steroids had unfavorable outcomes $(P=0.04)$ [17]. However, that study did not adjust for the severity of illness at admission, and no difference was observed between patients who received steroids and patients who did not receive any steroids. In our study population including all clinical subtypes of IAE, after adjusting for several factors such as patient characteristics, status at presentation, and therapeutic interventions, we did not observe effectiveness of early steroid pulse therapy. However, patients with some clinical subtypes of IAE may have benefit from the steroid pulse therapy $[16,17]$. Therefore, further studies are needed in this regard.

The present study had some limitations. First, this was a retrospective study, and all the data relied on past records in the database. Additionally, we only assessed the Japanese database; therefore, the generalizability of the results is limited. Second, we excluded patients who were transferred from and to other hospitals. Third, data on laboratory results such as higher creatinine $[18,19]$,

Table 3 Patient outcomes between propensity score-matched patients with and without steroid pulse therapy

\begin{tabular}{llll}
\hline & With steroid pulse therapy & Without steroid pulse therapy \\
& $N=168$ & $N=168$ & $P$ value \\
\hline Unfavorable outcome $^{a}, n(\%)$ & $23(13.7)$ & $14(8.3)$ & 0.16 \\
In-hospital mortality, $n(\%)$ & $1(0.6)$ & $2(1.2)$ & 1.0 \\
\hline
\end{tabular}

a Unfavorable composite outcome included (1) Japan Coma Scale $\geq 10$ at discharge, (2) requiring tracheostomy during hospital stay, (3) requiring mechanical ventilation at discharge, (4) requiring enteral tube feeding at discharge, (5) requiring rehabilitation at discharge, and (6) in-hospital death 
lactate dehydrogenase $[18,30]$, serum aspartate aminotransferase, alanine aminotransferase $[19,30]$, and lower platelet counts [18] were not available, although they may have been associated with patient outcomes. Similarly, we did not include results for electroencephalography or brain imaging such as computerized tomography and magnetic resonance imaging, which may have influenced the initiation for steroid pulse therapy. Fourth, IAE has several subtypes, which have different prognoses including AESD, ANE, and MERS [8] for which information was not available in the database. Nonetheless, we believe our results are important because identification of specific clinical subtype of IAE is sometimes difficult, especially in the early phase when steroid pulse therapy is thought to be beneficial $[18,19]$. Furthermore, although the ICD-10 code of F058 (delirium due to other medical condition) may have included mild IAE cases who did not benefit from the early steroid pulse therapy, the sensitivity analysis that excluded patients with the F058 code showed the same results. Fifth, although we adjusted for potential risk factors such as requiring mechanical ventilation, vasoactive agents, or admission to an intensive care unit, we did not include validated risk adjustment tools in our analyses. Sixth, our composite outcome was not validated. Although validated outcome measures such as Pediatric Cerebral Performance Category (PCPC) scale [31] may be warranted, they were not available in the database. Nonetheless, because the PCPC scale includes patient consciousness or coma status, we included JCS scores, which are thought to be correlated with the Glasgow Coma Scale scores, in our composite outcome [32]. Seventh, we did not identify long-term patient outcomes. A previous study reported that moderate or severe disability was maintained; however, mild disability may have improved thereafter [33].

\section{Conclusions}

In our retrospective observational study, we did not observe a significant difference in the proportions of unfavorable outcomes or in-hospital mortality between the patients with and without early steroid pulse therapy within 2 days of admission among children with IAE as a whole. However, further studies considering severity of illness are warranted to determine whether steroid pulse therapy is beneficial especially for specific clinical subtypes of IAE.

\section{Supplementary information}

Supplementary information accompanies this paper at https://doi.org/10. 1186/s40560-020-00479-8.

Additional file 1.

\section{Abbreviations}

IAE: Influenza virus-associated encephalopathy; MERS: Clinically mild encephalitis/encephalopathy with a reversible splenial lesion; AESD: Acute encephalopathy with biphasic seizures and late reduced diffusion;

ANE: Acute necrotizing encephalopathy; ICD-10: International classification of diseases 10th revision; JCS: Japan coma scale; Cl: Confidence interval

\section{Acknowledgements \\ Not applicable}

\section{Authors' contributions}

$\mathrm{TH}, \mathrm{NM}, \mathrm{MT}$, and HY contributed to the conception and design of this study; TH, NM, MT, HM., KF, and HY collected and analyzed data; TH drafted the manuscript; NM, MT, HM, KF, and HY critically reviewed the manuscript and supervised the whole study process. The author(s) read and approved the final manuscript.

\section{Funding}

This work was supported by grants from the Ministry of Health, Labour and Welfare, Japan (19AA2007 to H.Y. and H30-Policy-Designated-004 to H.Y. and K.F.) and the Ministry of Education, Culture, Sports, Science and Technology, Japan (17H04141 to H.Y.).

\section{Availability of data and materials}

The datasets generated and analyzed during the current study are not publicly available complying with data-sharing policies of the database.

\section{Ethics approval and consent to participate}

This study was approved by the Institutional Review Board at The University of Tokyo (Number: 3501-(3)). Given the anonymous nature of the database, informed consent was waived for this study.

\section{Consent for publication}

Not applicable

\section{Competing interests}

The authors declare that they have no competing interests.

\section{Author details}

'Department of Intensive Care Medicine, Osaka Women's and Children's Hospital, 840 Murodocho, Osaka, Izumi 594-1101, Japan. ²Department of Health Services Research, Graduate School of Medicine, The University of Tokyo, Tokyo, Japan. ${ }^{3}$ Department of Clinical Epidemiology and Health Economics, School of Public Health, The University of Tokyo, Tokyo, Japan. ${ }^{4}$ Department of Health Policy and Informatics, Tokyo Medical and Dental University Graduate School of Medicine, Tokyo, Japan.

Received: 28 April 2020 Accepted: 6 August 2020

Published online: 12 August 2020

\section{References}

1. Mizuguchi M, Yamanouchi H, Ichiyama T, Shiomi M. Acute encephalopathy associated with influenza and other viral infections. Acta Neurol Scand. 2007;115:45-56.

2. Kasai T, Togashi T, Morishima T. Encephalopathy associated with influenza epidemics. Lancet. 2000;355:1558-9.

3. Kedia S, Stroud B, Parsons J, Schreiner T, Curtis DJ, Bagdure D, et al. Pediatric neurological complications of 2009 pandemic influenza A (H1N1). Arch Neurol. 2011:68:455-62.

4. Amin R, Ford-Jones E, Richardson SE, MacGregor D, Tellier R, Heurter H, et al. Acute childhood encephalitis and encephalopathy associated with influenza: a prospective 11-year review. Pediatr Infect Dis J. 2008;27:390-5.

5. Goenka A, Michael BD, Ledger E, Hart IJ, Absoud M, Chow G, et al. Neurological manifestations of influenza infection in children and adults: results of a National British Surveillance Study. Clin Infect Dis. 2014;58:775-84.

6. Britton PN, Dale RC, Blyth CC, Macartney K, Crawford NW, Marshall H, et al. Influenza-associated encephalitis/encephalopathy identified by the Australian Childhood Encephalitis Study 2013-2015. Pediatr Infect Dis J. 2017;36:1021-6.

7. Mak CM, Lam C, Fong N, Siu W, Lee HH, Siu T, et al. Fatal viral infectionassociated encephalopathy in two Chinese boys: a genetically determined 
risk factor of thermolabile carnitine palmitoyltransferase II variants. J Hum Genet. 2011;56:617-21.

8. Hoshino A, Saitoh M, Oka A, Okumura A, Kubota M, Saito Y, et al. Epidemiology of acute encephalopathy in Japan, with emphasis on the association of viruses and syndromes. Brain Dev. 2012;34:337-43.

9. Gu Y, Shimada T, Yasui Y, Tada Y, Kaku M, Okabe N. National surveillance of influenza-associated encephalopathy in Japan over six years, before and during the 2009-2010 influenza pandemic. PLoS One. 2013;8:e54786.

10. Okuno H, Yahata Y, Tanaka-Taya K, Arai S, Satoh H, Morino S, et al. Characteristics and outcomes of influenza-associated encephalopathy cases among children and adults in Japan, 2010-2015. Clin Infect Dis. 2018;66: $1831-7$

11. Grose $C$. The puzzling picture of acute necrotizing encephalopathy after influenza A and B virus infection in young children. Pediatr Infect Dis J. 2004;23:253-4.

12. Ichiyama T, Isumi H, Ozawa H, Matsubara T, Morishima T, Furukawa S. Cerebrospinal fluid and serum levels of cytokines and soluble tumor necrosis factor receptor in influenza virus-associated encephalopathy. Scand J Infect Dis. 2003;35:59-61.

13. Sun G, Ota C, Kitaoka S, Chiba Y, Takayanagi M, Kitamura T, et al. Elevated serum levels of neutrophil elastase in patients with influenza virusassociated encephalopathy. J Neurol Sci. 2015;349:190-5.

14. Aiba H, Mochizuki M, Kimura M, Hojo H. Predictive value of serum interleukin-6 level in influenza virus-associated encephalopathy. Neurology. 2001;57:295-9.

15. Kobayashi Y, Togashi T, Mizuguchi M, Miyazaki C, Ichiyama T, Kawashima N, et al. The specific therapy for influenza encephalopathy in Japan. J Jpn Pediatr Soc. 2007;111:659-65 Article in Japanese.

16. Morishima T. Guideline for the management of influenza associated encephalopathy. Nihon Rinsho. 2006;64:1897-905 Article in Japanese.

17. Okumura A, Mizuguchi M, Kidokoro H, Tanaka M, Abe S, Hosoya M, et al. Outcome of acute necrotizing encephalopathy in relation to treatment with corticosteroids and gammaglobulin. Brain Dev. 2009;31:221-7.

18. Azuma J, Nabatame S, Nakano S, Iwatani Y, Kitai Y, Tominaga K, et al. Prognostic factors for acute encephalopathy with bright tree appearance. Brain Dev. 2015:37:191-9.

19. Yokochi T, Takeuchi T, Mukai J, Akita Y, Nagai K, Obu K, et al. Prediction of acute encephalopathy with biphasic seizures and late reduced diffusion in patients with febrile status epilepticus. Brain Dev. 2016;38:217-24.

20. Yamana H, Moriwaki M, Horiguchi H, Kodan M, Fushimi K, Yasunaga H. Validity of diagnoses, procedures, and laboratory data in Japanese administrative data. J Epidemiol. 2017:27:476-82.

21. Shigematsu K, Nakano H, Watanabe $\mathrm{Y}$. The eye response test alone is sufficient to predict stroke outcome-reintroduction of Japan Coma Scale: a cohort study. BMJ Open. 2013;3.

22. Rosenbaum PR, Rubin DB. Constructing a control group using multivariate matched sampling methods that incorporate the propensity score. Am Stat. 1985;39:33-8.

23. Austin PC. Balance diagnostics for comparing the distribution of baseline covariates between treatment groups in propensity-score matched samples. Stat Med. 2009:28:3083-107.

24. von Elm E, Altman DG, Egger M, Pocock SJ, Gøtzsche PC, Vandenbroucke $J$ J. et al. The Strengthening the Reporting of Observational Studies in Epidemiology (STROBE) statement: guidelines for reporting observational studies. Lancet. 2007:370:1453-7.

25. Morishima T, Togashi T, Yokota S, Okuno Y, Miyazaki C, Tashiro M, et al. Encephalitis and encephalopathy associated with an influenza epidemic in Japan. Clin Infect Dis. 2002;35:512-7.

26. Wang GF, Li W, Li K. Acute encephalopathy and encephalitis caused by influenza virus infection. Curr Opin Neurol. 2010;23:305-11.

27. Ito $\mathrm{Y}$, Ichiyama T, Kimura H, Shibata M, Ishiwada N, Kuroki H, et al. Detection of influenza virus RNA by reverse transcription-PCR and proinflammatory cytokines in influenza-virus-associated encephalopathy. J Med Virol. 1999;58:420-5.

28. Full F, Gack MU. Prostaglandin E2: the villain in the host response to influenza virus. Immunity. 2014;40:453-4.

29. Sellers SA, Hagan RS, Hayden FG, Fischer WA. The hidden burden of influenza: a review of the extra-pulmonary complications of influenza infection. Influenza Other Respir Viruses. 2017;11:372-93.

30. Motojima Y, Nagura M, Asano Y, Arakawa H, Takada E, Sakurai Y, et al. Diagnostic and prognostic factors for acute encephalopathy. Pediatr Int. 2016;58:1188-92
31. Fiser DH. Assessing the outcome of pediatric intensive care. J Pediatr. 1992; 121:68-74.

32. Todo T, Usui M, Takakura K. Treatment of severe intraventricular hemorrhage by intraventricular infusion of urokinase. J Neurosurg. 1991;74: $81-6$.

33. Nishiyama M, Nagase $H$, Tanaka T, Fujita K, Kusumoto M, Kajihara S, et al. Short and long-term outcomes in children with suspected acute encephalopathy. Brain Dev. 2016;38:731-7.

\section{Publisher's Note}

Springer Nature remains neutral with regard to jurisdictional claims in published maps and institutional affiliations.

\section{Ready to submit your research? Choose BMC and benefit from:}

- fast, convenient online submission

- thorough peer review by experienced researchers in your field

- rapid publication on acceptance

- support for research data, including large and complex data types

- gold Open Access which fosters wider collaboration and increased citations

- maximum visibility for your research: over $100 \mathrm{M}$ website views per year

At $\mathrm{BMC}$, research is always in progress.

Learn more biomedcentral.com/submissions 